\title{
Plasma Wave Propagation with Light Emission in a Long Positive Column Discharge
}

\author{
Guangsup $\mathrm{Cho}^{1}$ and John V. Verboncoeur ${ }^{2}$ \\ ${ }^{1}$ Department of Electrophysics, Kwangwoon University 447-1 Nowon Wallgye, \\ Seoul 139-701 \\ 2Department of Nuclear Engineering, University of California, Berkeley CA 94720, \\ ${ }^{1}$ Korea \\ ${ }^{2} U S A$
}

\section{Introduction}

The fine-tube cold cathode fluorescent lamp (CCFL) [1-2] and external electrode fluorescent lamp (EEFL) [3-9], have been widely used as a backlight source in liquid crystal display (LCD) modules. In a notebook PC, the glass tube diameter of the lamp is a small as $1 \mathrm{~mm}$. As the display area becomes larger in LCD TVs, the tube diameter is $3 \sim 4 \mathrm{~mm}$ and the lamp length is up to $1 \sim 2 \mathrm{~m}$. However, the mechanism for radiation of light along such a long positive column is not well understood.

The characteristics of light emission in the EEFLs have been reported, specifically the propagation of a light-emitting along a tube driven by high voltage AC [8-9]. In the long positive column lamp, the light emits first from the side of high voltage propagates to the ground side with the propagation time of $\sim \mu \mathrm{s}$ and the propagation velocity of $10^{5} \sim 10^{6} \mathrm{~m} / \mathrm{s}$. The previous observations have not been reported elsewhere to our knowledge since the fluorescent lamps have been introduced commercially in the 1940's.

However, those observations of light propagation would not be recognized even if they have been found first in the history of fluorescent lamps. Even more, those observations are misapprehended as the kinds of unstable waves during the lamp operation of lamp, such as striation, snaking, flickering, and streaming, etc. Generally, a moving light source in the long positive column plasma has been associated with "striation". A striation is a quasistanding wave which can be observed visually [10-12]. However, striations as well as the other unstable waves are not observed in commercial lamps, and would be considered a major defect for hot cathode fluorescent tubes as well as CCFL. In the manufacture of those lamps, inadequate vacuum during the evacuation process results in impurity gases such as $\mathrm{H}_{2} \mathrm{O}, \mathrm{CO}_{2}, \mathrm{O}_{2}$, and $\mathrm{N}_{2}$, etc. These impurities cause the perturbations of unstable discharge, and those lamps are discarded in the production line. Striations can sometimes appear in aging lamps or in the operation with an unstable inverter system. However, we have never observed striations in the normally manufactured lamps of our test samples. Our observation of optical signal is quite different from striations, as it is not visible to the naked eye and moves on the micro-second timescale. Rather than the instability, they are related to the stable process of distribution of plasma and fields that generate light in these discharges. 
In the previous studies, the propagation velocity of light-emitting wave-front along the positive column has been observed $u_{p} \sim\left(10^{+5} \sim 10^{+6}\right) \mathrm{m} / \mathrm{s}$. In Ref. [8], the propagation speed $u_{p}$ was explained as the electron drift velocity, $u_{p} \sim u_{d}$. However, typical electron drift velocities driven by an electric field are $u_{d} \leq 10^{+4} \mathrm{~m} / \mathrm{s}$ in a typical glow discharge plasma. Therefore, the propagation velocity of light emission cannot be related to the electron drift velocity. In Ref. [9], the propagation velocity has been explained as the electron plasma wave propagation having an electron thermal speed $u_{p} \sim u_{e}$. However, the electron plasma wave has not been described completely whether the wave can propagate a long distance of about 100 1,000 $\mathrm{mm}$ and a long time of a few $\mu$ s since the damping time of an electron plasma wave is very short, about $10^{-9} \mathrm{~s}$. In those respects, the propagation of optical emission could be neither the electron drift nor the electron plasma wave of electron thermal speed.

In this study, the propagation of light emission along a fine tube CCFL will be observed through the experimental measurement of the optical signals. CCFL can be operated with DC-voltage as well as AC-voltage while EEFL is driven only with AC-voltage. Through the operation of CCFL with DC and DC-ripple voltage, the propagation of light emission will be verified as new phenomena in a positive column discharge. The mechanism of these phenomena is the other scope in this study. However, the phenomena of light-propagation will provide a clue for the understanding of positive column plasma and a new motivation for the study of lamp physics. In this respect it is necessary to observe this phenomena more precisely again.

In Sec. 2, optical signal measurements are conducted according to the voltage of DC, rippled-DC, and AC in the operation of CCFLs. In the sub-section of Sec. 2-1, the optical propagation with DC-voltage operation is represented. In Sec. 2-2 with AC-voltage operation in the CCFL without coating the phosphor, the optical signal measurements are conducted according to the voltage variations. In this experiment we have obtained two different types of light propagation along the positive column. The propagation of light emission is analyzed with the plasma wave perturbation theory in Sec. 3. Discussions and conclusions are in Sec. 4 and Sec. 5, respectively.

\section{Observation of light emission along the positive column}

For the observation of light propagation along the tube, two kinds of operation as DC and AC voltage are involved. The DC-voltage operation with rippled voltage is the first attempt to observe the optical signals in the experiment of Sec. 2-1. In the AC-voltage operation of Sec. 2-2, we try to measure the light signals radiating directly from the discharge plasma through the naked glass without coating a phosphor inside glass wall. Additionally, the variation of optical signal according to the lamp current is represented in the AC-operation.

\section{2-1. DC-Operation}

In this experiment, the CCFL discharge tube is borosilicate glass, $0.5 \mathrm{~mm}$ thick with $4.0 \mathrm{~mm}$ outer diameter, and $699 \mathrm{~mm}$ length for 30" backlight units. The gas composition is typical for CCFLs, filled with $95 \%$ neon and $5 \%$ argon, at a total pressure of 60 Torr, and also containing $2 \mathrm{mg}$ of mercury in liquid phase. The inner surface of the glass tube is coated with RGB tri-phosphor. Cylindrical Ni-electrodes are inserted at the ends sealed with the connection of the lead line of electrode. 
The circuit system in this study has been designed to measure the optical signal with the conventional operation method as shown in Figure 1. For the operation of CCFL, two kinds of power supply systems are used as DC power and modulate-DC power as shown in the schematic drawing of the experimental circuit system. For DC and modulate-DC operation, a high voltage is applied to one end electrode connected with a $200 \mathrm{k} \Omega$ ballast resistor in series. The other electrode of lamp is grounded. The modulate-DC voltage is rippled $\pm \Delta V$ on the DC voltage $V_{\mathrm{o}}$ as $V(t)=V_{\mathrm{o}}+\Delta V \sin \omega t$. The lamp voltage is measured at the electrode side of high voltage. The lamp current is measured at the grounded electrode with ammeter. The optical signals are measured at three equally spaced points, (1) (3), along the tube surface with a photo-sensor, which is a light-to-voltage conversion amplifier using an optical fiber. The spacing between the measuring points is $\Delta L=305 \mathrm{~mm}$ and the length between (1) and (3) is $L=610 \mathrm{~mm}$ along the entire tube of $699 \mathrm{~mm}$ length.

Figure 2 for the operation with DC-voltage, shows the oscilloscope signals of voltage, current, and optical intensity measured at point (1) where the luminance is $10,000 \mathrm{~cd} / \mathrm{m}^{2}$. The bottom line is the base value of zero for the DC-voltage, the DCcurrent, and the optical intensity. The lamp voltage noted with solid line is DC $1.24 \mathrm{kV}$ after the voltage drops by $8.40 \mathrm{kV}$ at the $200 \mathrm{k} \Omega$ ballast resistor from the DC $9.64 \mathrm{kV}$ supplied at the DC-power source. The lamp current measured at the grounded electrode is $4.2 \mathrm{~mA}$. The lamp power is $5.2 \mathrm{~W}$. The optical signal as well as the current signal is constant on time due to the DC-voltage operation mode as it is expected. In the DC-voltage mode, the propagation of the optical signal cannot be observed since all the signals of light emission along the tube are the same. Since the light originates from atomic excitation due to the impact of high-energy electrons, the uniform light emission signals along the tube do not show any difference in the DCvoltage operation.

In order to observe the propagation of light-emission, a modulate-DC voltage is applied. The signals of voltage, current, and optical pulse, are represented with solid lines in Fig. 3(a). All the signals of straight line in the DC operation of Fig. 2 are also noted with dashed lines. The optical signal is measured at point (1) where the luminance is $10,000 \mathrm{~cd} / \mathrm{m}^{2}$. Using the switching diode in the circuit of power supply, the high AC-voltage of $64.5 \mathrm{kHz}$ is rectified to be a rippled high DC-voltage, $V_{\mathrm{o}}+\Delta V \sin \omega t$ with the ripple frequency $f=\omega / 2 \pi=129$ $\mathrm{kHz}$. The lamp voltage of rippled DC is averaged $V_{\mathrm{o}}=1.21 \mathrm{kV}$, while the output voltage from power source is $9.61 \mathrm{kV}$, so the voltage drops by $8.40 \mathrm{kV}$ at the $200 \mathrm{k} \Omega$ ballast resistor. The ripple voltage is $\Delta V=110 \mathrm{~V}$ with $\Delta V / V_{\mathrm{o}} \sim 10 \%$. Lamp current is also rippling as $I_{\mathrm{o}}+\Delta I \sin \omega t$ with $I_{\mathrm{o}} \sim 4.2 \mathrm{~mA}$ and $\Delta I \sim 1.1 \mathrm{~mA}$. The average lamp power is $5.1 \mathrm{~W}$. The optical signal observed at position (1) shows the wave rippled with the optical intensity of $O(t) \sim O_{\mathrm{o}}+\Delta O \sin \omega t$ with $\Delta O / O_{\mathrm{o}} \sim 10 \%$ and the same frequency of $129 \mathrm{kHz}$ as the rippling voltage and current.

In order to obtain the information of light-emission propagation for the operation of rippled DC voltage, the optical signals are observed at different points along the tube. Figure 3(b) shows the optical signals at points (1) (3) with the time delay for each signal. The optical signal at (1) is measured when the luminance is $10,000 \mathrm{~cd} / \mathrm{m}^{2}$ at (1). When the luminance at (1) is $10,000 \mathrm{~cd} / \mathrm{m}^{2}$, the luminance at (2) and (3) is slightly low as $9,600 \mathrm{~cd} / \mathrm{m}^{2}$ at (2), and $9,200 \mathrm{~cd} / \mathrm{m}^{2}$ at (3). The optical intensity at each measuring point is proportional to the luminance. When the optical intensity at (1) is averaged to be $O_{o}(1) \sim 1$, the intensity at (2) and (3) is to be decayed to be $O_{\mathrm{o}}$ (2) $\sim 0.96$ and $O_{\mathrm{o}}(3) \sim 0.92$, respectively. The time interval of optical signals between (1) (3) is the propagation time $t_{p}$ of light emission from (1) to (3). The propagation 
time is $t_{p}=1.82 \mu \mathrm{s}$. If the optical intensity from (1) to (3) is decayed as $O_{\mathrm{o}}(t)=\mathrm{O}_{\mathrm{o}} \exp \left(t / t_{D}\right)$, the characteristic decay time $t_{D}$ of optical intensity from (1) to (3) is $t_{D} \sim 22 \mu \mathrm{s}$ from the calculation of $O_{\mathrm{o}}(3) / O_{\mathrm{o}}(1) \sim 0.92 \sim \exp \left(-t_{p} / t_{D}\right)$ with $t_{p}=1.82 \mu \mathrm{s}$. The decay time $t_{D} \sim 22 \mu \mathrm{s}$ is longer than the optical propagation time $t_{p}=1.82 \mu \mathrm{s}$ as well as the period of ripple voltage, $t_{f}=1 / f=7.75 \mu \mathrm{s}$ with $f=129 \mathrm{kHz}$. The propagation speed of light emission is $u_{p}=3.35 \times 10^{5} \mathrm{~m} / \mathrm{s}$ which is calculated with $u_{p}=L / t_{p}$ as the ratio of the length between (1) (3) of $L=610 \mathrm{~mm}$ to the signal propagation time of $t_{p}=1.82 \mu \mathrm{s}$. If the operation voltage increases, the luminance as well as the light emission propagation speed increases.

\subsection{AC-operation of CCFL without phosphor coating}

In order to directly observe a light emission from the plasma discharge with CCFLs, the inner wall of the glass tube is not coated with phosphor. The CCFL discharge tube is borosilicate glass, $0.5 \mathrm{~mm}$ thick with $3.4 \mathrm{~mm}$ outer diameter, and $900 \mathrm{~mm}$ length. The gas composition is typical for CCFLs, filled with $95 \%$ neon and $5 \%$ argon, at a total pressure of 60 Torr, and also containing $2 m g$ of mercury in liquid phase.

The circuit system in this study has been designed to measure the optical signal with the conventional operation method as shown in Figure 4. The high voltage of $V(t)=V_{\mathrm{o}} \sin \left(\omega_{0} t\right)$ with $\omega_{0}=2 \pi f_{\mathrm{o}}$ is applied to one electrode through the ballast capacitor $\mathrm{C}=22 \mathrm{pF}$, and the other electrode is grounded. AC power is supplied through a DC-AC inverter with driving frequency $f_{\mathrm{o}}=65 \mathrm{kHz}$ with the period $15 \mu \mathrm{s}$. The optical signals are measured at equally spaced points, (1) (5), along the tube surface with a photosensor, which is a light-to-voltage conversion amplifier using an optical fiber. The spacing between the measuring points is $\Delta L=220 \mathrm{~mm}$ and the length between (1) and (5) is $L=880 \mathrm{~mm}$ along the entire tube of $900 \mathrm{~mm}$ length. The lamp current is measured at the ground, and the circuit voltage $V_{a}$ is measured at the point a and the lamp voltage $V_{b}$ is at the point $b$.

Figure 5 shows the I-V curves represented with the applied voltage $V_{a}$ and the lamp voltage $V_{b}$. It shows the typical glow discharge process, the primary discharge before Townsend breakdown, and the normal glow discharge after Townsend breakdown. As the circuit voltage $V_{a}$ is increased, the current of the primary discharge increases to a few $m A$. It increases to $2.6 \mathrm{~mA}$ just before the breakdown voltage $V_{a}=2 \mathrm{kV}$, and it increases abruptly to be several tens of $m A$ after breakdown. However, the lamp voltage $V_{b}$ is increased to be 1.8 $k V$ at Townsend breakdown and the voltage falls to below $0.98 k V$ after Townsend breakdown. The curve of lamp current versus lamp voltage $V_{b}$ shows the property of negative resistance after Townsend breakdown. In this experiment, the optical signals are measured at the low current range noted with Figs. 6(a) and 6(b) before Townsend breakdown and at the high current of Figs. 6(c) and 6(d) after Townsend breakdown.

Figure 6 shows the oscilloscope signals measured at points (1) (5) when the CCFL driving voltage is varied. The applied voltage and current corresponding to Figs. $6(\mathrm{a}) \sim 6(\mathrm{~d})$ are noted in the I-V curve of Fig. 5. Fig. 6(a) is the applied voltage $V_{a}=1.8 \mathrm{kV}$, the lamp voltage $V_{b}=1.6$ $k V$, and the lamp current $2.5 \mathrm{~mA}$. Fig. $6(\mathrm{~b})$ is just before Townsend breakdown with $V_{a}=2.0$ $k V, V_{b}=1.8 \mathrm{kV}$, and the lamp current $2.6 \mathrm{~mA}$. Fig. 6(c) is just after Townsend breakdown with $V_{a}=2.3 \mathrm{kV}, V_{b}=0.98 \mathrm{kV}$, and $I=31.0 \mathrm{~mA}$. Fig. $6(\mathrm{~d})$ is $V_{a}=2.45 \mathrm{kV}, V_{b}=0.97 \mathrm{kV}$, and $I=32.6 \mathrm{~mA}$. All the voltage and current data are in root mean square values. Since the peaks of the optical signals correspond to the voltage peaks of $\pm V_{\mathrm{o}}$ in two polarities, the frequency of the optical signal on the oscilloscope is $2 f_{\mathrm{o}}=130 \mathrm{kHz}$, twice that of the driving voltage. 
Figs. 6 show the optical signals of the discharges before and after Townsend breakdown. In Figs. 6(a) and 6(b) before Townsend breakdown, the optical signals have a time shift and a decay of intensity as the measuring point goes from (1) to (5). The optical intensity corresponding to the negative polarity of voltage is a little higher than that of the positive polarity at the same measuring point. In Figs. 6(c) and 6(d) after Townsend breakdown, the time shifts of each optical signal measured at the points (1) (5) are smaller and the deviations of optical intensity at each point and for both polarities are also smaller than those of Figs. $6(\mathrm{a})$ and 6(b). However, we have two types of optical signals. For the first type, the propagation of the optical signal is slow and the optical intensity decays as the measuring point from (1) to (5) as shown in Figs. 6(a) and 6(b). For the second type, the propagation time is short and the intensity is nearly the same value between measuring points as shown in Figs. 6(c) and 6(d).

In the optical signals of Fig. 6(a), the total propagation time from (1) to (5) of $L=880 \mathrm{~mm}$ length is $t_{p} \sim 5.31 \mu \mathrm{s}$ with the mean propagation velocity $u_{p} \sim L / t_{p} \sim 1.7 \times 10^{+5} \mathrm{~m} / \mathrm{s}$. The propagation speed slows slightly from (1) to (5) but is nearly constant. In the optical signals of Fig. 6(b), the propagation time from (1) to (5) is $t_{p} \sim 4.00 \mu s$ with the mean propagation velocity $u_{p} \sim 2.2 \times 10^{+5} \mathrm{~m} / \mathrm{s}$. As the current and voltage increase before breakdown, the propagation speed $u_{p}$ increases. After the breakdown discharge of Figs. 6(c) and 6(d), the propagation speed is $u_{p} \sim 2.6 \times 10^{+6} \mathrm{~m} / \mathrm{s}$ with the propagation time $t_{p} \sim 0.34 \mu \mathrm{s}$ in Fig. 6(c) and the propagation speed is $u_{p} \sim 4.6 \times 10^{+6} \mathrm{~m} / \mathrm{s}$ with $t_{p} \sim 0.19 \mu \mathrm{s}$ in Fig. 6(d). The propagation speed after breakdown is faster by a factor of 10 .

For the explanation of the optical propagation according to the two types of discharge, before and after the Townsend breakdown, the plasma diffusion and the electron plasma wave theory is adopted in the positive column plasma. In plasma diffusion, we calculate the diffusion speed along the discharge tube from the diffusion equation. For the electron plasma wave, the driving frequency $f_{\mathrm{o}} \sim 65 \mathrm{kHz}$ is treated as a source of the perturbation into the positive column plasma of the normal glow discharge after the Townsend breakdown. The key issues of this study will be whether a plasma wave of this driving frequency can be propagated, and the physical effects that cause this wave to be propagated through the positive column filled with high density plasma.

\section{Analysis of plasma propagation along the positive column}

For the purpose of understanding the propagation of light emission, the experimental observations in the previous sections are summarized as:

i. Light always propagates from the high voltage side to the ground. The direction of propagation is independent of the electron drift velocity $u_{d}$ whose directions are varied according to the electric field with the polarity of applied voltage.

ii. The speed of light propagation is $u_{p} \sim\left(10^{5}-10^{6}\right) \mathrm{m} / \mathrm{s}$ according to the lamp current. At the low current $\sim 1 \mathrm{~mA}$, the velocity is $u_{p} \sim 10^{5} \mathrm{~m} / \mathrm{s}$. As the current increases at the normal glow, the propagation velocity also increases over $10^{6} \mathrm{~m} / \mathrm{s}$.

iii. The optical signals represent the type of ripple wave as $O(t)=O_{\mathrm{o}}+d O \sin \omega t$. The ripple frequency is the frequency of voltage pulses. In DC-operation, the ripple frequency is the frequency of DC-voltage pulse. When the operation frequency is $\omega_{\mathrm{o}}$ in AC-voltage, the frequency of optical ripple wave is $\omega=2 \omega_{0}$, 2-times of AC-operation frequency. With respect to the ripple ratio $d O / O_{\mathrm{o}}$, we have two types of optical signals according to the 
current. At the low current before breakdown, the ripple ratio can be very large over 50 $\%$ and the signals decay along the $z$-direction of positive column as $O(t, z)=O_{\mathrm{o}}+d O \exp (-$ $\left.z / z_{D}\right) \sin \omega t$. The scale of decay is about the lamp length $L$ as $z_{D} \sim L$. At the normal glow of high current, the optical signals would not represent the decay with the large scale of decay as $z_{D}>L$ and the ripple ratio is about $d O / O_{\mathrm{o}} \sim 20-30 \%$.

For the interpretation of experimental results summarized above, our models for the analysis are listed below as:

i. Plasma is generated at high voltage side of $z=0$ and diffused toward the ground electrode of $z=L$. At the steady state, the gradient of plasma density is formulated by the continuous generation of plasma at the high voltage side. The density is high at $z=0$ and is decreased toward $z=L$.

ii. Perturbation is generated at the high voltage side by the high voltage $V(t)=V_{\mathrm{o}} \sin \omega_{\mathrm{o}} t$ with the frequency $f_{0}=\omega_{0} / 2 \pi$. The wave is generated initially at the high voltage side with the frequency $\omega=2 \pi\left(2 f_{\mathrm{o}}\right) \sim 10^{5} \mathrm{~s}^{-1}$ for the operation frequency $f_{\mathrm{o}}=65 \mathrm{kHz}$. In this analysis we will show the wave always propagates to the $z$-direction with the propagation velocity and the decay. We will find what kinds of effect are for the propagation.

iii. The wave cannot be observed with the naked eye. It means the wave length is submillimeter. Then the phase velocity is determined with the wave frequency $\omega \sim 10^{5} \mathrm{~s}^{-1}$. The group velocity of propagation should be $\sim\left(10^{5}-10^{6}\right) \mathrm{m} / \mathrm{s}$ according to the experimental data. For the presentation of wave decay along the tube, we have the characteristic decay length, which should be above the scale of lamp length, about $1 \mathrm{~m}$ or $10 \mathrm{~m}$ according to the lamp current.

In the previous reports [8-9], the fatal results of their analysis are that the waves are dependent of the electron drift velocity $u_{d}$ whose direction is changed with the voltage polarity. Therefore, they do not explain the direction of propagation observed in the experiments where the waves always propagate from high voltage side to the ground without decay or with a small decay.

\subsection{Electron plasma fluid equations and plasma parameters}

The electron plasma waves have been described in Ref. [13-22] where all the waves cannot be propagated due to the high damping due to the elastic collision of electron. For the difference from Ref. [8-19], this report includes plasma density gradient along the positive column, which will be an important factor for the propagation of electron plasma waves without damping. The electron equation of motion and continuity equation are with $J \approx-n e u_{d}$ :

$$
\begin{gathered}
n\left[\frac{\partial \vec{u}}{\partial t}+(\vec{u} \cdot \vec{\nabla}) \vec{u}\right]=-v_{e}\left(\vec{J} / e+D_{a} \vec{\nabla} n+n \vec{u}\right) \\
\frac{\partial n}{\partial t}+\vec{\nabla} \cdot(n \vec{u})=v_{i z} n
\end{gathered}
$$

In the above electron fluid equation, the ambi-polar diffusion is considered [14-19]. Before manipulating the equations, the plasma parameters in this study are estimated follows.

In the general fluorescent lamps for LCD-TVs, the typical gas composition is filled with $\mathrm{Ne}$ $(95 \%)+\operatorname{Ar}(5 \%)$, at a total pressure 60 Torr, and also containing $2 m g$ of mercury in liquid 
phase. The neutral atom density is $n_{n} \sim 10^{+24} \mathrm{~m}^{-3}$ from the calculation of $n_{n} \sim 3.25 \times 10^{+22} \mathrm{P}$ (Torr) $m^{-3}$ [13-14]. The plasma is mostly Hg-ions with the plasma density $n_{0} \sim\left(10^{+16} \sim 10^{+17}\right) \mathrm{m}^{-3}$ and the electron plasma temperature $T_{e} \sim 1 \mathrm{eV}$ in a typical positive column of $\mathrm{Hg}$ discharge. The mean electron energy is $\varepsilon=3 T_{e} / 2 \sim 1.5 \mathrm{eV}$ and the electron thermal velocity is $u_{e}=(2 \varepsilon / m)^{1 / 2} \sim 7 \times 10^{+5} \mathrm{~m} / \mathrm{s}$. An electron collision frequency is $v_{e} \sim n_{n} \sigma u_{e} \sim 2.3 \times 10^{+10} \mathrm{~s}^{-1}$ with $\sigma(\mathrm{Ne}) \sim 2 \times 10^{-20} \mathrm{~m}^{2}$ at $\varepsilon=1.5 \mathrm{eV}$ [23]. The electron mean free path is $\lambda_{e} \sim\left(1 / n_{n} \sigma\right) \sim 43 \mu \mathrm{m}$. The ionization collision frequency is $v_{i z} \sim n_{n} \sigma^{i z} \mathcal{U}_{e} \sim 10^{+6} \mathrm{~s}^{-1}$ where the ionization collision cross section is very sensitive to the value of electron thermal energy $T_{e}$.

Generally, the diffusion coefficient of electron, ambi-polar, and ion, are estimated to be $D_{e} \sim 10^{+1}>D_{a} \sim\left(10^{-2} \sim 10^{-3}\right)>D_{i} \sim 10^{-5}\left(\mathrm{~m}^{2} / \mathrm{s}\right)$. In the calculation of $D_{e}$, we obtain $D_{e} \sim 35 \mathrm{~m}^{2} / \mathrm{s}$ with $T_{e}=1 \mathrm{eV}$ and $v_{e} \sim 10^{+10} \mathrm{~s}^{-1}$. When the mercury ion mass $(\mathrm{Hg}) \sim 200 \mathrm{~m}_{p}$ (proton mass) and the ion

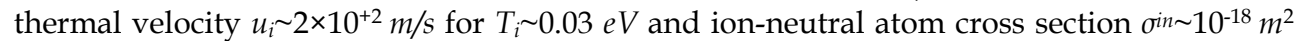
[15], we have the ion-neutral atom collision frequency $v_{i}=n_{n} \sigma^{i n} \mathcal{u}_{i} \sim 4 \times 10^{+8} \mathrm{~s}^{-1}$. Then, we obtain $D_{a}=T_{e} / M v_{i} \sim 2.6 \times 10^{-3} \mathrm{~m}^{2} / \mathrm{s}$.

The plasma density $n_{\mathrm{o}}$ is verified and the electron drift velocity $u_{d}$ is calculated with the experiment data. When the operation frequency is $f_{\mathrm{o}}=60 \mathrm{kHz}$, the lamp current is about $1 \mathrm{~mA}$ or less before breakdown and the current is about $10 \mathrm{~mA}$ for the normal glow after breakdown. From the current $i=d Q / d t \sim Q f_{\mathrm{o}}$, we have the total charge $Q=i / f_{\mathrm{o}} \sim\{(1-$ 10) $\left./\left(60 \times 10^{3}\right)\right\} \times 10^{-7} \mathrm{C}$ corresponding to the current $i=(1-10) m A$. Then, the total plasma particles are $N=Q / e$. The plasma density is $n_{\mathrm{o}}=N /\left(\pi r_{\mathrm{o}}{ }^{2} \mathrm{~L}\right) \sim\left(1.4 \times 10^{16} \sim 1.4 \times 10^{17}\right) \mathrm{m}^{-3}$ with the tube inner radius $r_{\mathrm{o}} \sim 1.5 \times 10^{-3} \mathrm{~m}$ and the tube length $L \sim 1 \mathrm{~m}$. The typical glow discharge plasma density is verified to be $n_{\mathrm{o}} \sim 10^{16} \mathrm{~m}^{-3}$ before breakdown for the low current $i \sim 1 \mathrm{~mA}$ and to be $n_{\mathrm{o}} \sim 10^{17} \mathrm{~m}^{-3}$ after breakdown for the current $\sim 10 \mathrm{~mA}$ for the normal glow plasma. With the plasma density $n_{\mathrm{o}}$, the electron drift velocity is calculated from $J=n_{\mathrm{o}} e u_{d}=i / A$ and the tube cross section $A=\pi r_{0}{ }^{2} \sim 7 \times 10^{-6} \mathrm{~m}^{2}$. Before breakdown for the low current $i \sim 1 \mathrm{~mA}$ and $n_{\mathrm{o}} \sim 1.4 \times 10^{16} \mathrm{~m}^{-3}$, we have $u_{d} \sim 5 \times 10^{4} \mathrm{~m} / \mathrm{s}$. Even the plasma after breakdown for $i \sim 10 \mathrm{~mA}$ and $n_{\mathrm{o}} \sim 1.4 \times 10^{17} \mathrm{~m}^{-3}$, we also have the electron drift velocity $u_{d} \sim 5 \times 10^{4} \mathrm{~m} / \mathrm{s}$. The typical value of drift velocity is known to be $u_{d} \sim 10^{4} \mathrm{~m} / \mathrm{s}$.

\subsection{Plasma diffusion along the tube}

For the analysis of the experimental results, plasma diffusion is considered in a fine tube positive column. When a high voltage is applied to the electrode, the plasma of high density is generated at the high voltage electrode, and the electrons and ions diffuse along the positive column. Even if electron and ion fluxes are made up of identical ambi-polar diffusion fluxes and drift fluxes due to the electric current driven by the external field $E$, we have the diffusion equation for each plasma species of electrons and ions including the plasma generation in the continuity equation with the ionization collision $v_{i z}$ [13-14]:

$$
\left(\frac{\partial n}{\partial t}\right)-D_{a} \nabla^{2} n=v_{i z} n
$$

The motion of a charged particle is considered with radial and longitudinal components. Considering diffusion with time dependence in the $z$-direction only for a bounded plasma within the tube of inner radius $r_{0}$, the solution can be written $n(t, z, r)=n_{r}(r) n_{z}(t, z)$. The radial component of Bessel's equation of order zero becomes $\nabla_{r}^{2} n_{r}+\left(c_{0} / D_{a}\right) n_{r}=0$ with a constant co 
coupling to the $z$-component. The solution to the radial component is the Bessel function of order zero, $n_{r}(r) \sim J_{\mathrm{o}}\left(r /\left[D_{d} / c_{\mathrm{o}}\right]^{1 / 2}\right)$. To satisfy the boundary condition $n_{r}\left(r_{\mathrm{o}}\right)=0$ at the tube radius $r_{0}$, we have $\alpha=r_{\mathrm{o}} /\left[D_{d} / \mathcal{C}_{\mathrm{o}}\right]^{1 / 2}$ which is the zero of the Bessel function $J_{\mathrm{o}}(\alpha)=0$. The coupling constant $c_{\mathrm{o}}=D_{a}\left(\alpha / r_{\mathrm{o}}\right)^{2}$ depends on the value of $\alpha$. According to the diffusion mode in a cylindrical cross section [13-19], the first zero of $J_{\mathrm{o}}$ is at $\alpha=2.4$.

Considering the diffusion only in the z-direction, we obtain the continuity equation

$$
\frac{\partial n_{z}}{\partial t}-D_{a} \nabla_{z}^{2} n_{z}=-\left(\frac{\alpha}{r_{o}}\right)^{2} D_{a} n_{z}+v_{i z} n_{z}
$$

One of the solutions for Eq. (2) is the time dependant Einstein's diffusion equation [16-18]:

$$
n_{z}(t, z)=c t^{-1 / 2} e^{-\left(z^{2} / 4 D_{a} t\right)} e^{-\left(\alpha / r_{o}\right)^{2} D_{a} t} e^{v_{i t} t}
$$

where $\mathrm{c}$ is a normalization constant.

If the radial diffusion effect and ionization collisions are not included as $r_{\mathrm{o}}$ becomes infinite and $v_{i z}=0$, the solution becomes the well known solution of Einstein's diffusion equation [1618], which is one dimensional diffusion away from a point source in an infinite medium. The solution represents the spread of the Gaussian formulation with the $z$-function. As time increases, the density of $n_{z}(t, z)$ will decrease and the distribution tends to become increasingly uniform.

The diffusion velocity is calculated as $u_{n}=-D_{a}\left(\nabla_{z} n\right) / n=z / 2 t$. Using the approximation of $\left(\alpha / r_{\mathrm{o}}\right) 2 D_{a}>>v_{i z}$ and $z>>r_{\mathrm{o}}$ since the tube radius is $r_{\mathrm{o}}=1.2 \mathrm{~mm}$ and the positive column length is $0<z<1 \mathrm{~m}$ in this experiment, the time $t$ at the peak value of density is obtained directly from Eq. (5) with $d n / d t=0$ :

$$
t \sim \frac{r_{0}}{2 \alpha D_{a}} z
$$

The above relation of Eq. (5) can be simply checked again from the derivative of exponential terms as $\left(z^{2} / 4 D_{a} t\right)+\left(\alpha / r_{\mathrm{o}}\right) 2 D_{a} t$ in Eq. (4) with $\left(\alpha / r_{\mathrm{o}}\right) 2 D_{a}>>v_{i z}$ and $z>>r_{\mathrm{o}}$. Then we have $u_{n}=-$ $D_{a}\left(\nabla_{z} n\right) / n=\mathrm{z} / 2 \mathrm{t} \sim\left(\alpha / r_{\mathrm{o}}\right) D_{a} \sim 40 \mathrm{~m} / \mathrm{s}$ with $\alpha=2.4, r_{\mathrm{o}}=1.2 \times 10^{-3} \mathrm{~m}$, and $D_{a} \sim 10^{-2} \mathrm{~m}^{2} / \mathrm{s}$. In this section we verify the diffusion velocity $u_{n}$ due to the plasma density gradient along the long positive column. When a high voltage applied to the electrode with the frequency $\omega=4 \Pi f_{\mathrm{o}} \sim 10^{5} \mathrm{~s}^{-1}$, the plasma is generated at the high voltage side and it diffuses very slowly and travels only about $0.1 \mathrm{~mm}$ in the period about $10 \mu \mathrm{s}$ of voltage pulse. Therefore, the plasma density gradient is formulated along the positive column in the steady state, a high density at the high voltage side and a low density at the ground side.

\subsection{Electron plasma waves}

The electron plasma wave [13-22] is represented with the perturbations of the wave varying as $f_{1}=f_{1} \exp [i(k z-\omega t)]$ where $k$ is the propagation vector in the $z$-direction. The electron density $n$, the electron plasma fluid velocity $\vec{u}$, and the electric field $\vec{E}$, are separated into two parts: an equilibrium part indicated by the subscript $\mathrm{o}$, and a perturbation part indicated by a subscript 1: $n=n_{0}+n_{1}, \vec{u}=\vec{u}_{0}+\vec{u}_{1}, \vec{E}=\vec{E}_{0}+\vec{E}_{1}$. 
The equilibrium values of plasma are obtained at the steady state of electron fluid. When $d \vec{u}_{0} / d t \sim 0$ in the force balance of Eq. (1), the electron plasma fluid velocity $\vec{u}_{0}$ is represented

$$
\vec{u}_{0}=-\frac{\vec{J}}{n_{0} e}-D_{a} \frac{\vec{\nabla} n_{0}}{n_{0}}=\vec{u}_{d}+\vec{u}_{n}
$$

The electron drift velocity is $\vec{u}_{d}=-\mu_{e} \vec{E}_{0}=-J /\left(n_{0}\right) e$ in the electric field $\vec{E}_{0}$. The electron fluid diffusion velocity of density gradient is $\vec{u}_{n}=-D_{a} \nabla n_{\mathrm{o}} / n_{\mathrm{o}}$.

Considering the plasma density in the continuity equation with steady state of $\partial n_{0} / \partial t \sim 0$, we have $\nabla^{2} n_{\mathrm{o}}+\left(v_{i z} / D_{a}\right) n_{\mathrm{o}}=0$. The plasma is generated at $z=0$ by the high voltage applied to the electrode and diffused into the positive column to $\mathrm{z}=\mathrm{L}$ of lamp length. One of the solution $n_{\mathrm{o}}$ $(\mathrm{z})=n_{\mathrm{o}} \cos \left\{\left(v_{i z} / D_{a}\right)^{1 / 2} z\right\}$. The corresponding diffusion velocity is $u_{n}=\left(v_{i z} D_{a}\right)^{1 / 2} \tan \left\{\left(v_{i z} / D_{a}\right)^{1 / 2} z\right\}$. The averaged magnitude of diffusion velocity is estimated to be $u_{n} \sim\left(v_{i z} D_{a}\right)^{1 / 2} \sim 10^{2} \mathrm{~m} / \mathrm{s}$ with $D_{a} \sim 10^{-2} \mathrm{~m}^{2} / \mathrm{s}$ and $v_{i z} \sim 10^{6} \mathrm{~s}^{-1}$. The diffusion velocity $\sim 10^{2} \mathrm{~m} / \mathrm{s}$ is too slow to diffuse into long positive column within micro-second of voltage pulse duration time so that the plasma diffusion signal is not fitted to the velocity of optical signal propagation having $10^{5} \sim 10^{6} \mathrm{~m} / \mathrm{s}$ in this experiments. It takes about $10^{-2} \mathrm{~s}$ diffusing along the long positive column of $1 \mathrm{~m}$ length with this diffusion velocity. The distance of diffusion is about $0.6 \mathrm{~mm}$ during the period $\sim 7 \mu$ s of a high voltage pulse.

The time var iation of equilibrium quantities are $d \vec{u}_{0} / d t \sim 0, \partial n_{0} / \partial t=0$. The spatial variation of equilibrium plasma density is considered as $\nabla n_{\mathrm{o}}=-\left(n_{0} / D_{a}\right) \vec{u}_{n}$ which provides the diffusion velocity $\vec{u}_{n}$ in this analyses. If we assume the velocity $u_{\mathrm{o}}$ as a constant value along the positive column for the simplicity, we have $\nabla u_{\mathrm{o}}=0$. When we consider $\nabla u_{\mathrm{o}}=0$, this effect are not affected on our results which will be discussed later.

Using the equilibrium values as $d \vec{u}_{0} / d t \sim 0, \partial n_{\mathrm{o}} / \partial t=0, \nabla u_{\mathrm{o}}=0$, and $\nabla n_{\mathrm{o}}=-\left(n_{\mathrm{o}} / D_{a}\right) \vec{u}_{n}$, the perturbed equations of $u_{1}$ and $n_{1}$ are

$$
\begin{gathered}
n_{0}\left(\frac{\partial u_{1}}{\partial t}+u_{0} \nabla u_{1}\right)=-v_{e}\left[\frac{J_{1}}{e}+D \vec{\nabla} n_{1}+n_{1} \vec{u}_{0}+n_{0} u_{1}\right] \\
\frac{\partial n_{1}}{\partial t}+\vec{u}_{0} \cdot \vec{\nabla} n_{1}+n_{0} \vec{\nabla} \cdot \vec{u}_{1}+\vec{u}_{1} \cdot \vec{\nabla} n_{0}=v_{i z} n_{1}
\end{gathered}
$$

Using $J_{1} /\left(n_{0}\right) e=\left(-\vec{u}_{d}\right)_{1} \approx \mu_{e} \vec{E}_{1}=-\mu_{e} \vec{\nabla} V_{1}=-i k \mu_{e} V_{1}=i \mu_{e} e n_{1} /\left(\varepsilon_{0} k\right)$ and $\nabla^{2} V_{1} \approx e n_{1} / \varepsilon_{0}$, we have $-k^{2} V_{1}=e n_{1} / \varepsilon_{0}$ by the assumption of stationary ions. Using $\partial / \partial t=-i \omega$ and $\nabla=i k$, the perturbed equations are

$$
\begin{gathered}
n_{0} u_{1}\left(-i \omega+i k u_{0}+v_{e}\right)=-n_{1} v_{e}\left[i \mu_{e} \frac{n_{0} e}{\varepsilon k}+i k D+u_{0}\right] \\
n_{1}\left(-i \omega+i k u_{0}-v_{i z}\right)=-n_{0} u_{1}\left(i k-\frac{u_{n}}{D_{a}}\right)
\end{gathered}
$$


Combining above two equations, the dispersion equation of the electron plasma waves is obtained by the factoring out the first-order quantities:

$$
\omega^{2}-\omega_{p e}^{2}-\kappa u_{e}^{2} k^{2}-2 \omega k u_{o}+i \omega v_{e}-i k u_{n} v_{e}-i \frac{u_{n} v_{e}}{\kappa \lambda_{D}{ }^{2} k}-\frac{u_{n} u_{o} v_{e}{ }^{2}}{\kappa u_{e}{ }^{2}}=0
$$

In the above equation, we use $\omega_{p e}{ }^{2} \gg v_{e} v_{\text {iz }}$ and $v_{e} D_{a} \gg u_{\mathrm{o}}^{2}$ since $v_{e} D_{a}=\kappa u_{e}{ }^{2}$ with $\kappa=m v_{e} / M v_{i}$ and the relation $\lambda_{D} \omega_{p e}=u_{e}$ with the Debye shielding length $\lambda_{D}$.

If the electron flow $u_{0}=u_{d}+u_{n}$ and the electron collision frequencies $v_{e}$ and $v_{i z}$ are ignored, we have the typical electron plasma wave as the oscillation electrons at $\omega_{p e}$ are streaming into adjacent layers of plasma with thermal velocities $u_{e}$ [14-19].

With an explicit expression for $\omega=\omega_{r}+i \omega_{i}$, the imaginary part of $\omega$ is expressed as

$$
\omega_{i}=-\frac{v_{e}}{2} \frac{\omega_{r}-k u_{n}\left(1+1 / \kappa \lambda_{D}^{2} k^{2}\right)}{\omega_{r}-k u_{o}}
$$

For the propagation wave without damping as $\omega_{i}=0$, we have the wave,

$$
\omega_{r}=k u_{n}\left(1+1 / \kappa \lambda_{D}{ }^{2} k^{2}\right)
$$

For the general electron plasma waves without density gradient as $\nabla n_{\mathrm{o}}=0$ and $u_{n}=0$ in the above equation, we can verify all the waves are fast damping with the damping frequency $\omega_{i} \sim-v_{e} / 2 \sim-10^{-10} s^{-1}$. The wave frequency $\omega_{r}$ is independent of the drift velocity $u_{d}$ whose direction is changed by the polarities of applied voltage.

For the description of wave propagation along the longitudinal direction $\mathrm{z}$ of positive column, corresponding to the optical signals observed in this experiment, we would rather take the explicit expression with $k=k_{r}+i k_{i}$ than $\omega=\omega_{r}+i \omega_{i}$. The imaginary $\omega_{i}<0$ shows the time decay as $\exp \left(\omega_{i} \mathrm{t}\right)$ while the imaginary of $k_{i}>0$ represents the scale of decay in the $z$-direction as $\exp (i k z)=\exp \left(i k_{r} z\right) \exp \left(-k_{i} z\right)$. When the scale of decay in the $z$-direction is $z_{D}$, we have $k_{i}=1 / z_{D}$ for $\exp \left(-z / z_{D}\right)$. In this experiment the decay scale of $z_{D}$ is about $1 \sim 10 \mathrm{~m}$. In a low current of $\sim 1 \mathrm{~mA}$ we have $z_{D} \sim 1 \mathrm{~m}$ which is $k_{i} \sim 1$. In the normal glow plasma of current $\sim 10$ $m A$ we have a large decay scale as $z_{D} \sim 10 m$ which is the case of $k_{i} \sim 10^{-1}$.

With an explicit expression for $k=k_{r}+i k_{i}$, the imaginary part of $k$ is expressed as

$$
k_{i}=\frac{v_{e}}{2} \frac{\omega-k_{r} u_{n}\left(1+1 / \kappa \lambda_{D}{ }^{2} k_{r}^{2}\right)}{\omega u_{o}+\kappa k_{r} u_{e}^{2}}
$$

For the propagation wave without damping longitudinally with $k_{i}=0$ in the above equation, we have the same result obtained from $\omega_{i}=0$ in Eq. (13) as

$$
\omega=k_{r} u_{n}\left(1+1 / \kappa \lambda_{D}{ }^{2} k_{r}^{2}\right)
$$

The wave is approximated to be $\omega \sim u_{n} \lambda / 2 \pi \kappa \lambda_{D}{ }^{2} 10^{5} \mathrm{~s}^{-1}$ with $k_{r}=2 \pi / \lambda, u_{n} \sim 10^{2}, \kappa \sim 10^{-2}$. Then, we have the wave length $\lambda \sim 10\left(2 \pi \lambda_{D}^{2}\right) \sim 0.1 \mathrm{~mm}$.

The real part of $k$ is given by 


$$
\omega^{2}-\omega_{p e}^{2}-\kappa u_{e}^{2}\left(k_{r}^{2}-k_{i}^{2}\right)-2 \omega k_{r} u_{o}+k_{r} u_{n} v_{e}-\frac{k_{i}}{k_{r}{ }^{2}} \frac{u_{n} v_{e}}{\kappa \lambda_{D}{ }^{2}}-\frac{u_{n} u_{o} v_{e}{ }^{2}}{\kappa u_{e}{ }^{2}}=0
$$

From the derivative of $d \omega$ and $d k_{r}$ in the above equation of dispersion relation, the propagation velocity of wave is calculated in the group velocity $u_{p}=d \omega / d k_{r}$ with $\omega$ in Eq. (16) and $k_{i}=0$;

$$
u_{g}=\frac{d \omega}{d k_{r}}=\frac{u_{e}{ }^{2}+u_{o} u_{n} / \kappa\left(\lambda_{D} k_{r}\right)^{2}-u_{n} v_{e} / k_{r}}{u_{n} / \kappa\left(\lambda_{D} k_{r}\right)^{2}-u_{d}} \approx \frac{u_{e}{ }^{2}}{u_{n}} \kappa\left(\lambda_{D} k_{r}\right)^{2} \sim\left(10^{5}-10^{6}\right)(\mathrm{m} / \mathrm{s})
$$

In the approximation of above equation, we have $u_{e}>u_{n} / \kappa\left(\lambda_{D} k_{r}\right)^{2}>u_{d} \sim 10^{4}$ with $u_{n} \sim 10^{2}, \kappa \sim 10^{-2}$, $k_{r}=2 \pi / \lambda$, which is $\left(u_{e} / u_{n}\right)>\left(\lambda / 2 \pi \lambda_{D}\right)^{2} / \kappa>\left(u_{d} / u_{n}\right) \sim 10^{2}$. Using $\left(\lambda / 2 \pi \lambda_{D}\right)>10 \kappa^{1 / 2} \sim 1$ with $\lambda>2 \pi \lambda_{D}$, we have the wavelength of $\lambda \sim 0.1 \mathrm{~mm}$. Finally, the group velocity of wave propagation is $u_{g} \sim u_{e}{ }^{2} \kappa\left(2 \pi \lambda_{D} / \lambda\right)^{2} / u_{n} \sim 10^{6} \mathrm{~m} / \mathrm{s}$ or less with $u_{e} \leq 10^{6} \mathrm{~m} / \mathrm{s}$.

The wave corresponds to the optical signals as $O(t, z)=O_{0}+d O \sin \omega t$. The perturbed wave $f(t$, $z$ ) describes $d O$. We have the wave as $O(t, z)=O_{o}+d O \sin \left\{2 \pi\left(z u_{g} t\right) / \lambda_{g}\right\} \sin \left\{2 \pi\left(z-u_{p} t\right) / \lambda\right\} \exp \left(-k_{i} z\right)$. The frequency is $\omega=4 \pi f_{\mathrm{o}} \sim 130 \mathrm{kHz} \sim 10^{5} \mathrm{~s}^{-1}$. The wave length is $\lambda \sim 2 \pi \lambda_{D} \sim 0.1 \mathrm{~mm}$. The propagation velocity is the group velocity $u_{g}=d \omega / d k \sim 10^{6} \mathrm{~m} / \mathrm{s}$ of modulated wave whose wavelength is $\lambda_{\mathrm{g}} \sim 2 \pi \mathrm{m}$, the scale of lamp length. Since the value of $k_{i}$ can be zero or small enough for the wave to propagate along the whole length of lamp, the scale of decay along the longitudinal direction is $z_{D}=k_{i}^{-1}$ which can be larger than the lamp length.

\section{Discussions}

The experimental observations summarized in the beginning of previous section, are explained with the analysis of wave propagation as follows:

i. The wave always propagates along the tube from the high voltage side to the ground. The direction of propagation is independent of the electron drift velocity. The propagation of wave is in the direction of diffusion velocity $u_{n}$ since the group velocity $d \omega / d k \sim u_{e}{ }^{2} / u_{n}$ as well as the phase velocity $\omega / k \sim u_{n}$ is related to the diffusion velocity $u_{n}$. In the previous reports [8-9], the direction of wave has been represented in the direction of electron drift velocity $u_{d}$ as $\omega / k \sim u_{d}$ and $d \omega / d k \sim u_{e}^{2} / u_{d}$.

ii. In the observation, the light propagation speed is $u_{p} \sim 10^{5}-10^{6} \mathrm{~m} / \mathrm{s}$ according to the lamp current. At the low current of $\sim 1 \mathrm{~mA}$, the velocity is $u_{p} \sim 10^{5} \mathrm{~m} / \mathrm{s}$. As the current increases at the normal glow, the propagation velocity also increases over $10^{6} \mathrm{~m} / \mathrm{s}$. The propagation velocity in the analysis is the group velocity of $d \omega / d k \sim u_{e}^{2} / u_{n}$ which is proportional to the square of electron thermal velocity $u_{e}$. Generally in Townsend breakdown, the most important parameter in Townsend discharge is the electron energy $T_{e}=m u_{e}^{2} / 3$. For instance, in $\mathrm{Hg}$-discharge with rare gases, the electron temperature is $T_{e}<1 \mathrm{eV}$ before the breakdown and it can be $T_{e}>1 \mathrm{eV}$ at the normal glow and it can be increased with the discharge current.

iii. In the analysis, the wave describes the optical signals as $O(t, z)=O_{\mathrm{o}}+d O \exp \left(-z / z_{D}\right) \sin \omega t$. Since the propagation velocity corresponds to the group velocity, and it becomes fast to be $\sim 10^{6} \mathrm{~m} / \mathrm{s}$ as the current is increased, the wave would not decay for the short time of $\sim \mu \mathrm{s}$. If the propagation velocity is $\sim 10^{5} \mathrm{~m} / \mathrm{s}$, the propagation time of $\sim 5 \mu \mathrm{s}$ is long enough 
for the wave to be in decay. However, the scale of decay is the length of lamp even for the velocity $\sim 10^{5} \mathrm{~m} / \mathrm{s}$.

The mechanism of light propagation is explained with the results of analysis in the previous Sec. 3 as follows:

i. Plasma is generated at high voltage side of $z=0$ and diffused toward the ground electrode of $z=L$. At the steady state, the gradient of plasma density is formulated by the continuous generation of plasma at the high voltage side. The plasma density is high at $z=0$ and is decreased toward $z=L$. There are two kinds of electron flow in the fluid velocity $u_{0}$. One is the electron drift $u_{d} \sim 10^{4} \mathrm{~m} / \mathrm{s}$ driven by the electric field. Another is the electron diffusion velocity $u_{n}$ driven by the gradient of plasma density $\nabla n_{\mathrm{o}}$. The electron flow of diffusion with the velocity $u_{n} \sim 10^{2} \mathrm{~m} / \mathrm{s}$, due to the plasma density gradient in the steady state, is the origin of plasma wave propagation along the positive column.

ii. The wave is generated by the pulse of high voltage which is the role of the perturbation on the positive column plasma having a density gradient. Initially, the wave is generated at the high voltage side with the frequency of voltage pulse. The perturbed wave propagates along the slope of plasma density to the $z$-direction.

iii. The wavelength of wave is about ten times of Debye length as $\lambda \sim 10 \lambda_{D} \sim 0.1 \mathrm{~mm}$ so that it cannot be observed with the naked eye. The scale of modulated group wave is the scale of lamp length so that the group wave has no nodal point along the positive column. The wave of decay along the tube has the decay length above the scale of lamp length, about $1 \mathrm{~m}$ or $10 \mathrm{~m}$ according to the lamp current. Therefore, the wave seems to be a flowing tide, rising from ocean tiding toward beach.

\section{Conclusion}

The observation of light propagation along a long positive column is the first report in 70years history of long discharge lamps such as neon-sign lamps, fluorescent lamps for general lighting, and CCFLs and EEFLs for LCD-TVs. The mechanism of light propagation has not been understood even in the previous reports [8-9].

While the wave is not generated in the DC-operation of constant voltage, the wave is observed only in the operation with DC-rippled voltage. Through the operation of DCrippled voltage, the optical signals observed in this experiment are verified not to be a kind of unstable standing waves like a striation but it is clearly a new kind of wave propagating along the positive column.

In the AC-operation, the propagation velocity of light emission depends on the lamp current. At the low current before breakdown, the propagation velocity is about $10^{5} \mathrm{~m} / \mathrm{s}$ and the wave decays with the scale of lamp length. As the current increases after Townsend breakdown of normal glow, the propagation velocity is increased with the order of $10^{6} \mathrm{~m} / \mathrm{s}$. The wave of high current will not show the decay during propagation.

The origin of propagating wave in this observation is the electron diffusion wave. When the plasma is generated by the high field at the side of electrode applied a high voltage, the plasma diffuses very slowly toward the ground electrode. In the steady state, the gradient of plasma density is formulated along the positive column, a high density at the high voltage electrode and a low density at the ground electrode. When the perturbed wave is generated due to the high voltage pulses which roles a perturbation at the side of high voltage electrode, the wave slides on the slope of plasma density along the positive column and propagates toward the ground electrode. 
The substance of wave is the electron diffusion flow enforced by the plasma density gradient formulated along the whole positive column. The phase velocity as well as the group velocity of wave is proportional to the electron diffusion velocity. The wavelength of phase is a sub-millimeter so that the wave cannot be seen with naked eyes. Since the wavelength of modulated group wave is the scale of lamp length, the wave is propagating like a flowing tide rising from the high voltage electrode toward the grounded electrode.

\section{Acknowledgments}

This work was supported by Brain Korea 21 under a research grant in 2009.

\section{References}

1. J. Waymouth, Electrical Discharge Lamps, MIT Press, 1971.

2. R. Y. Pai, SID'97 Digest, 1997, p. 447.

3. T. S. Cho, Y. M. Kim, N. O. Kwon, S. J. Kang, E. H. Choi, and G. S. Cho, "Effect of Electrode Length on Capacitively Coupled External Electrode Fluorescent Lamp," Jpn. J. Appl., Phys., Vol. 41, No. 3B, 2002, pp. L355-L357.

4. T. S. Cho, H. S. Kim, Y. G. Kim, J. J. Ko, J. G. Kang, E. H. Choi, G. S. Cho, H. S. Uhm, "Characteristic Properties of Fluorescent Lamps Operated Using Capacitively Coupled Electrodes," Jpn, J. Appl. Phys., Vol. 41, No. 12, 2002, pp. 7518-7521.

5. T. S. Cho, N. O. Kwon, Y. M. Kim, H. S. Kim, S. J. Kim, J. G. Kang, E. H. Choi, and G. S. Cho, " Capacitive coupled electrodeless discharge backlight driven by square pulses", IEEE Trans. Plasma Sci., Vol. 30, No. 5, 2002, pp. 2005-2009.

6. G. S. Cho, N. O. Kim, Y. M. Kim. S. J. Kim, T. S. Cho, B. S. Kim, J. G. Kang, E. H. Choi, U. W. Lee, S. C. Yang, and H. S. Uhm, "Self-discharge Synchronizing Operations in the External Electrode Fluorescent Multi-lamps Backlight," J. Phys. D, Appl. Phys., Vol. 36, 2003, pp. 2526-2530.

7. G. S. Cho, M. J. Shin, J. M. Jeong, "Glass tube of high dielectric constant and low dielectric loss for external electrode fluorescent lamps.", Journal of Applied physics, Vol. 102, No. 11, 2007, p. 113307.

8. G. S. Cho, S. H. Ahn, J. M. Jeong, "Plasma propagation in external electrode fluorescent lamps." J. phys. D: Appl. Phys, Vol. 40, No. 13, 2007, pp. 39453950.

9. G. S. Cho, J. H. Kim, J. M. Jeong, “Electron plasma wave propagation in external electrode fluorescent lamps.", Applied Physics Letters, Vol. 92, No. 2, 2008, p. 021502.

10. A. Garscadden, D. A. Lee, "Forward and backward-wave moving striations in constricted discharge", Int. J. Electronic Control, Vol. 20, No. 6, 1966, p. 567.

11. D. A. Lee, P. Bletzinger, A. Garscadden, "Wave nature of moving striations", J. Appl. Phys., Vol. 37, No. 1, 1966, p. 377.

12. M. Hirsh and H. J. Oskam, "Gaseous electronics", Vol. 1, Electric Discharges, (Academic Press,1978), Part 2.2, p. 65.

13. Francis F. Chen, Introduction to Plasma Physics and Controlled Fusion, Vol. 1: Plasma Physics, 2nd ed. (Plenum, New York, 1984), Chap. 1, p. 13.

14. Y. Raizer, "Gas Discharge Physics", (Springer-Verlag Berlin Heidelberg 1991), Chap. 2, p. 32.

15. M. Lieberman and A. Lichtenberg, "Principles of Plasma Discharges and Materials Processing", (Wiley \& Sons, N.Y. 2005), Chap. 10, p. 334. 
16. E. Nasser, "Fundamentals of Gaseous Ionization and Plasma Electronics", (WileyInterscience, 1970), Chap. 6, p. 183.

17. E. Nasser, "Fundamentals of Gaseous Ionization and Plasma Electronics", (WileyInterscience, 1970), Chap. 4, p. 112, and Appendix D, p. 448.

18. L. Loeb, "Fundamental processes of Electrical Discharge in Gases", (John Wiley \& Sons, Inc., 1939), Chap. 4, p. 177.

19. A. von Engel, "Ionized Gases", 2nd ed. (Oxford University Press, 1965), Chap. 4, p. 122.

20. Blake E. Cherrington, "Gaseous Electronics and Gas Lasers", (Pergamon Press Inc. U.S.A., 1979), Chap. 8, p. 149.

21. N. A. Krall and A. W. Trivelpiece, "Principles of Plasma Physics", (McGraw-Hill, N. Y. 1973), Chap. 6.

22. B. Cherrington, "Gaseous Electronics and Gas Lasers", (Pergamon Press, 1979), Chap. 7, p. 116.

23. A. Salop and H. H. Nakano, Phys. Rev., A 2, p. 127, 1970. 


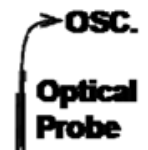

(1)

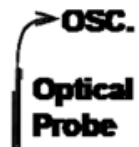

(2)

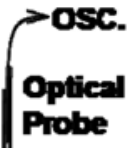

(3)

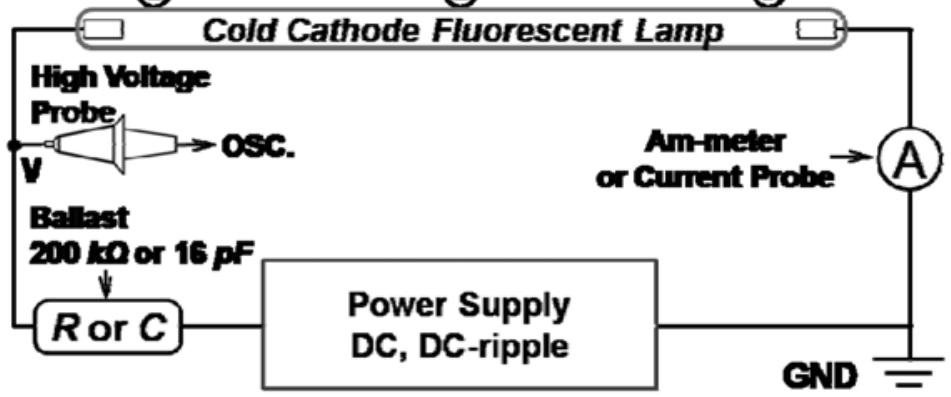

Fig. 1. Experimental circuit for measuring the optical signals in CCFL operated with DC or AC electric power supply. The high voltage is supplied to the left side of electrode and the other side is grounded. Measuring points of the optical signal are labeled with (1) (3).

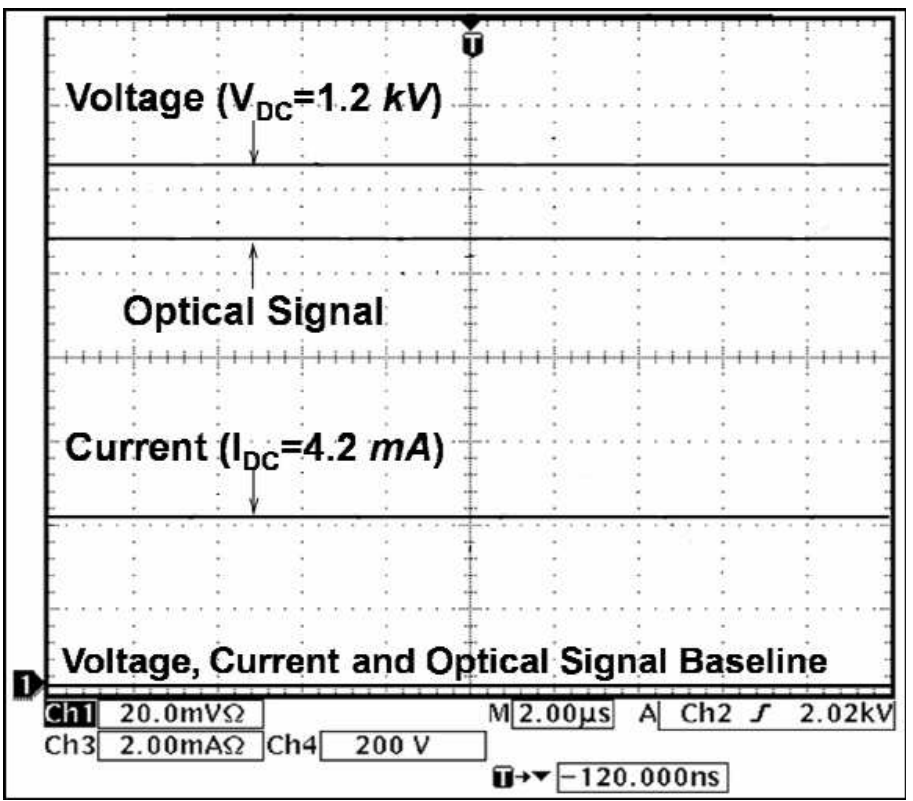

Fig. 2. Oscilloscope signals of lamp voltage, lamp current, and optical probe intensity for the operation with the DC voltage. The solid bottom line is the zero base line. All the signals are constant on time. 


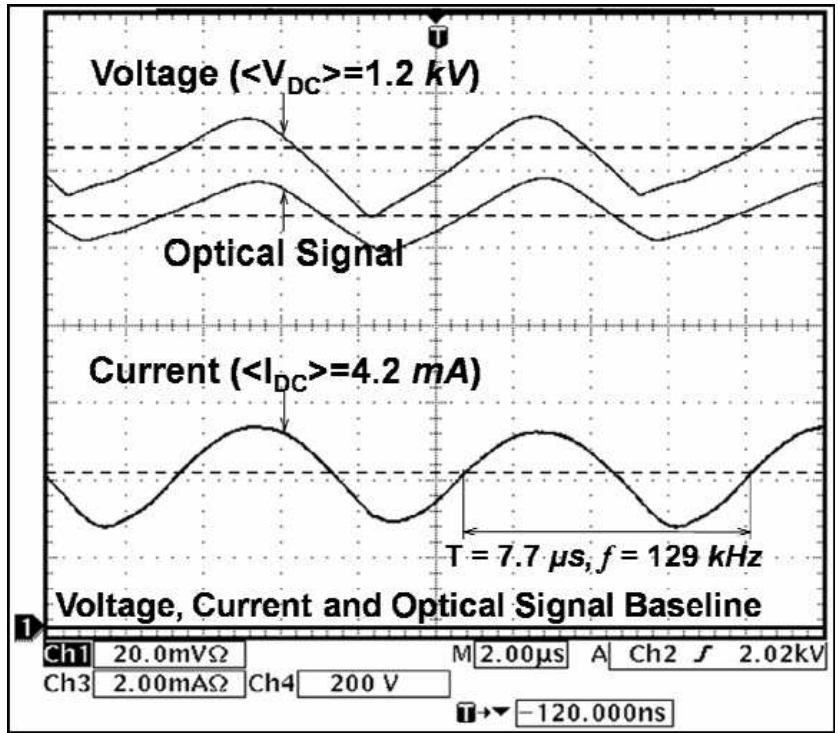

Fig. 3(a). Oscilloscope signals of lamp voltage $1.2 \mathrm{kV}$, lamp current $4.2 \mathrm{~mA}$, and optical probe intensity for the operation with the rippled-DC voltage as $V(t)=V_{\mathrm{o}}+\Delta V \sin \omega t$ with $\Delta V / V_{\mathrm{o}} \sim 10$ $\%$. The solid bottom line is the zero base line of voltage, current, and optical intensity. The straight dashed-lines are obtained from the DC operation in Fig. 2. All the signals show the rippled sinusoidal wave with the operation DC frequency $129 \mathrm{kHz}$ and the period of $7.7 \mu \mathrm{s}$. The optical intensity is $O(t) \sim O_{\mathrm{o}}+\Delta O \sin \omega t$ with $\Delta O / O_{\mathrm{o}} \sim 10 \%$.

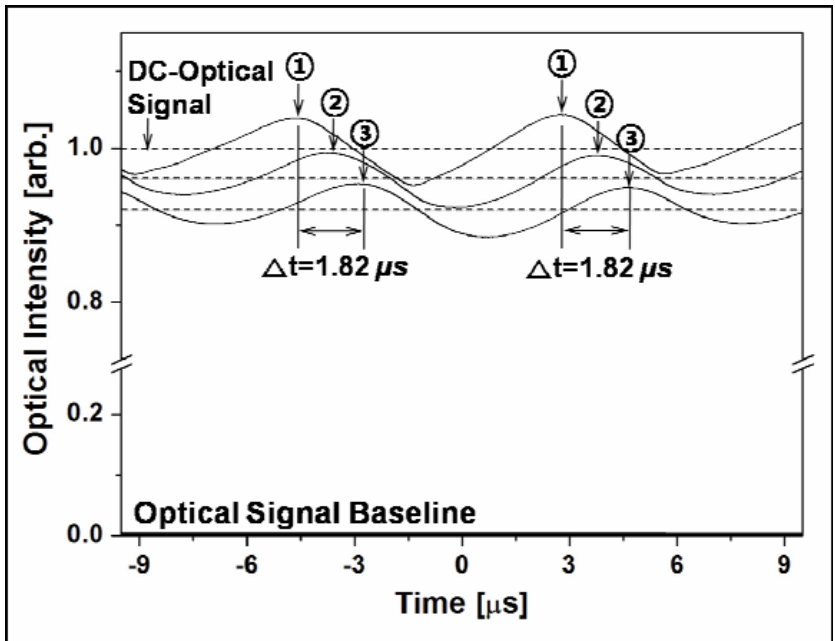

Fig. 3(b). The optical signals measured at points (1) (3) when the luminance at (1) is 10,000 $\mathrm{cd} / \mathrm{m}^{2}$ for the operation with the rippled-DC voltage as shown in Fig. 3(a). The propagation time of optical emission from (1) to (3) is $t_{p}=1.82 \mu \mathrm{s}$. The propagation velocity is $u_{p}=3.35 \times 10^{5}$ $\mathrm{m} / \mathrm{s}$ traveling the distance $610 \mathrm{~mm}$ from (1) to (3). During the propagation, the optical intensity decays slightly with the characteristic decay time $t_{D} \sim 22 \mu \mathrm{s}$. 


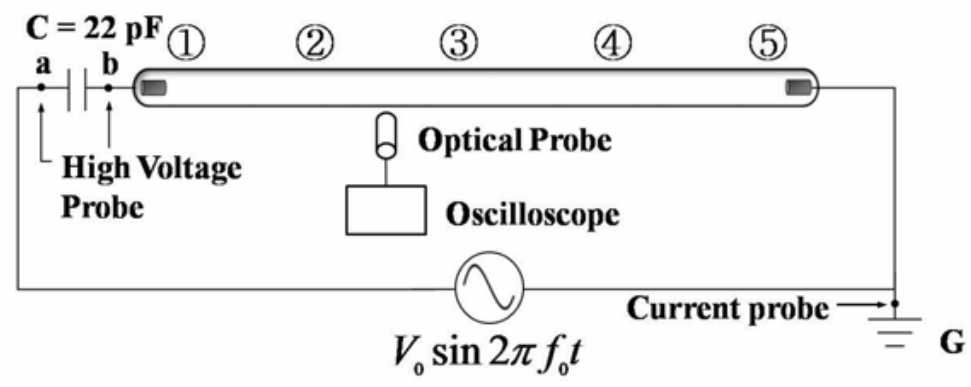

Fig. 4. Experimental circuit for measuring the optical signals at points (1) (5) along the CCFL. Lamp length is $900 \mathrm{~mm}$, with $220 \mathrm{~mm}$ between each point, and $880 \mathrm{~mm}$ between points of (1) and (5).

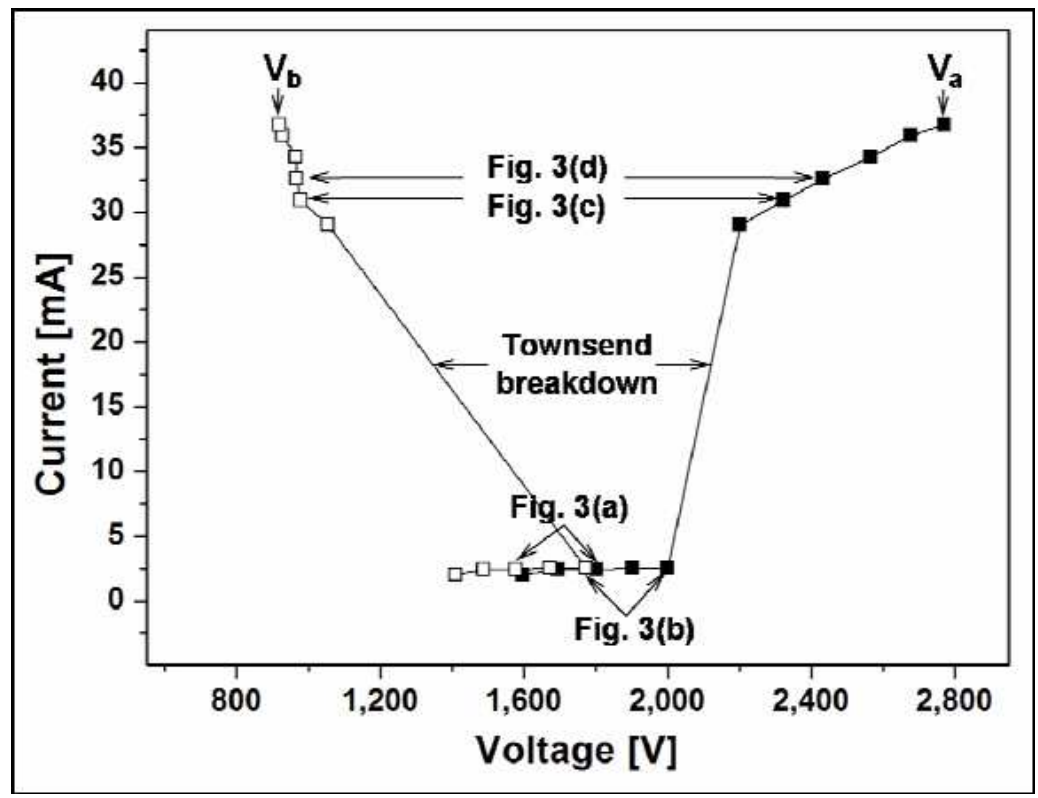

Fig. 5. Current and voltage curves with the applied voltage $V_{a}$ and the lamp voltage $V_{b}$ measured at the point $\mathrm{a}$ and $\mathrm{b}$ in the circuit of Fig. 1, respectively. The optical signals of Figs. 3 are measured at the following I-V: Fig. 3(a) is with $I=2.5 m A, V_{a}=1.8 \mathrm{kV}, V_{b}=1.6 \mathrm{kV}$, Fig. 3(b) is with $I=2.6 \mathrm{~mA}, V_{a}=2.0 \mathrm{kV}, V_{b}=1.8 \mathrm{kV}$, Fig. $3(\mathrm{c})$ is with $I=31.0 \mathrm{~mA}, V_{a}=2.32 \mathrm{kV}, V_{b}=0.98 \mathrm{kV}$, and Fig. $3(\mathrm{~d})$ is with $I=32.6 m A, V_{a}=2.45 \mathrm{kV}, V_{b}=0.97 \mathrm{kV}$, respectively. 
a) $2.5 \mathrm{~mA}, t_{p}=5.31 \mathrm{~ms}, t_{p}=1.66 \times 10^{5} \mathrm{~m} / \mathrm{s}$

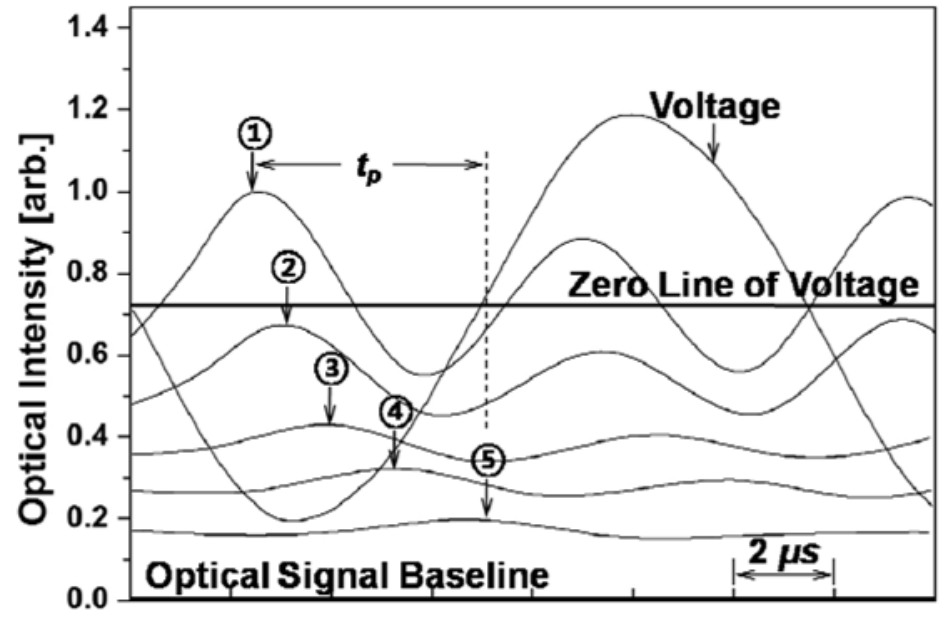

b) $2.6 \mathrm{~mA}, t_{p}=4.00 \mu \mathrm{s,} u_{p}=2.20 \times 10^{5} \mathrm{~m} / \mathrm{s}$

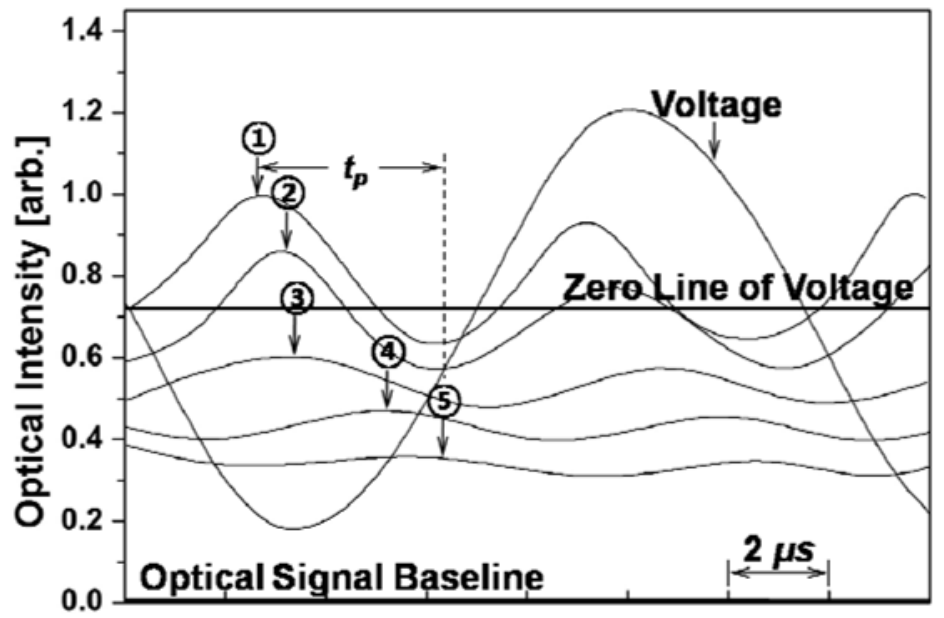


c) $31.0 \mathrm{~mA}, t_{p}=0.34 \mu \mathrm{s}, u_{p}=2.60 \times 10^{6} \mathrm{~m} / \mathrm{s}$

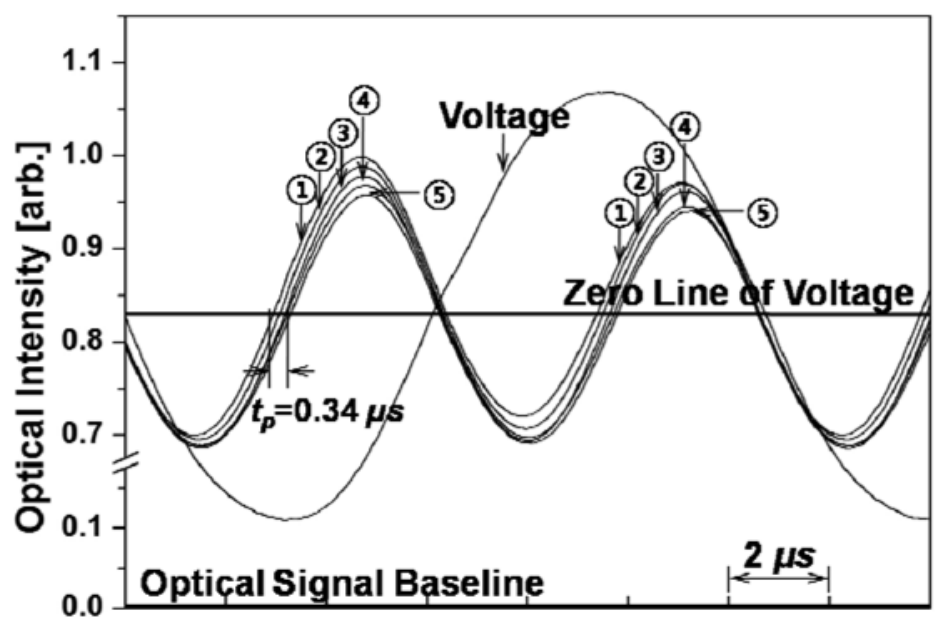

d) $32.6 \mathrm{~mA}, t_{p}=0.19 \mathrm{\mu s}, u_{p}=4.60 \times 10^{5} \mathrm{~m} / \mathrm{s}$

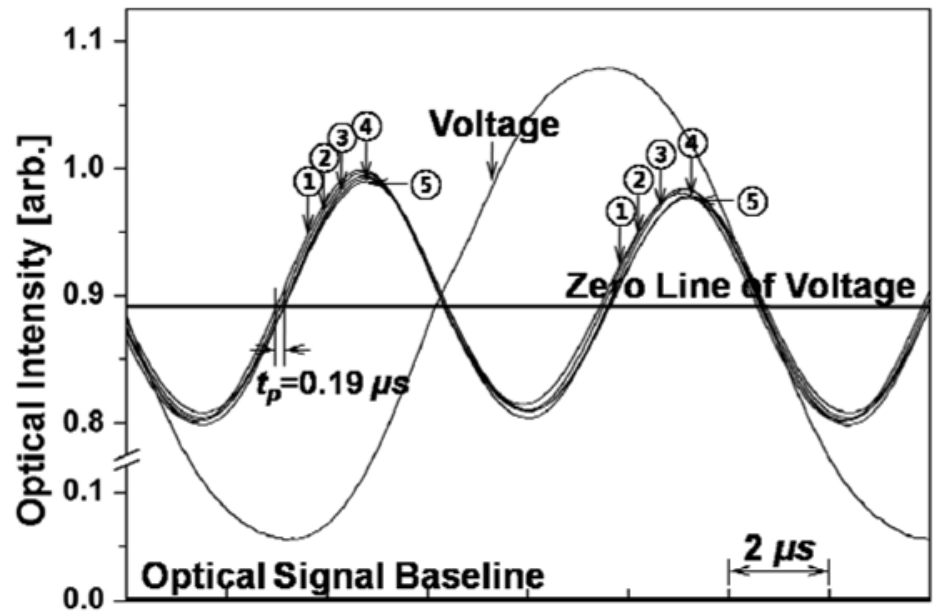

Fig. 6. Oscilloscope signals of the lamp voltage $V_{b}$ and the optical probe pulses measured at each point (1) (5). The signals at (a) and (b) are before Townsend breakdown and those at (c) and (d) are after Townsend breakdown as they are noted in Fig. 2. If the voltage signal is $V(t)=V_{\mathrm{o}} \sin \omega_{\mathrm{o}} t$ with $\omega_{\mathrm{o}}=2 \pi f_{\mathrm{o}}$ for the applied frequency $f_{\mathrm{o}}=65 \mathrm{kHz}$, then the corresponding optical signal is represented by $O(t)=O_{0}+O_{1} \sin \left\{\omega_{o p}(t-\Delta t)\right\}$ with the frequency of $\omega_{o p}=2 \omega_{\mathrm{o}}$ for the two polarities of voltage peaks $\pm V_{\mathrm{o}}$. In the optical signals at (a) and (b), the optical intensity $O_{o}$ decays remarkably with the measuring points from (1) to (5). The decay rate of 
optical signal from (1) to (5) is quite slow at (c) and (d). In the figures from (a) to (d), the propagation time $\Delta t$ of the optical signal from the peak of (1) to (5) becomes short so that the propagation velocity $u_{p}$ becomes fast. Before Townsend breakdown of low current $I=2.5 \mathrm{~mA}$ and $I=2.6 \mathrm{~mA}$ in figures (a) and (b), the propagation time from (1) to (5) is $5.31 \mu \mathrm{s}$ and $4.00 \mu \mathrm{s}$, and the mean propagation velocity is $u_{p}=880 \mathrm{~mm} / 5.31 \mu \mathrm{s}=1.66 \times 10^{5} \mathrm{~m} / \mathrm{s}$ at (a) and $u_{p}=2.20 \times 10^{5}$ $\mathrm{m} / \mathrm{s}$ at (b). After Townsend breakdown of high current $I=31.0 \mathrm{~mA}$ and $I=32.6 \mathrm{~mA}$ in figures (c) and (d), the propagation time from (1) to (5) is $0.34 \mu \mathrm{s}$ and $0.19 \mu \mathrm{s}$, and the mean propagation velocity is $u_{p}=2.60 \times 10^{6} \mathrm{~m} / \mathrm{s}$ at $(\mathrm{c})$ and $u_{p}=4.60 \times 10^{6} \mathrm{~m} / \mathrm{s}$ at (d). 


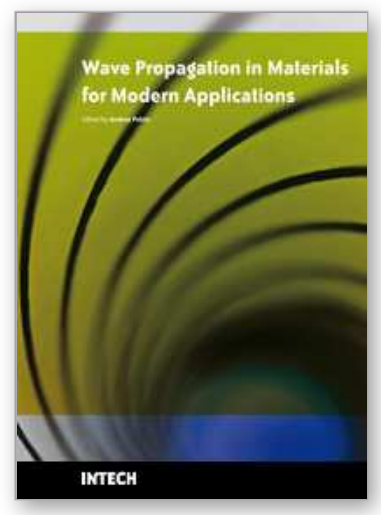

\section{Wave Propagation in Materials for Modern Applications}

Edited by Andrey Petrin

ISBN 978-953-7619-65-7

Hard cover, 526 pages

Publisher InTech

Published online 01, January, 2010

Published in print edition January, 2010

In the recent decades, there has been a growing interest in micro- and nanotechnology. The advances in nanotechnology give rise to new applications and new types of materials with unique electromagnetic and mechanical properties. This book is devoted to the modern methods in electrodynamics and acoustics, which have been developed to describe wave propagation in these modern materials and nanodevices. The book consists of original works of leading scientists in the field of wave propagation who produced new theoretical and experimental methods in the research field and obtained new and important results. The first part of the book consists of chapters with general mathematical methods and approaches to the problem of wave propagation. A special attention is attracted to the advanced numerical methods fruitfully applied in the field of wave propagation. The second part of the book is devoted to the problems of wave propagation in newly developed metamaterials, micro- and nanostructures and porous media. In this part the interested reader will find important and fundamental results on electromagnetic wave propagation in media with negative refraction index and electromagnetic imaging in devices based on the materials. The third part of the book is devoted to the problems of wave propagation in elastic and piezoelectric media. In the fourth part, the works on the problems of wave propagation in plasma are collected. The fifth, sixth and seventh parts are devoted to the problems of wave propagation in media with chemical reactions, in nonlinear and disperse media, respectively. And finally, in the eighth part of the book some experimental methods in wave propagations are considered. It is necessary to emphasize that this book is not a textbook. It is important that the results combined in it are taken "from the desks of researchers". Therefore, I am sure that in this book the interested and actively working readers (scientists, engineers and students) will find many interesting results and new ideas.

\section{How to reference}

In order to correctly reference this scholarly work, feel free to copy and paste the following:

Guangsup Cho and John V. Verboncoeur (2010). Plasma Wave Propagation with Light Emission in a Long Positive Column Discharge, Wave Propagation in Materials for Modern Applications, Andrey Petrin (Ed.), ISBN: 978-953-7619-65-7, InTech, Available from: http://www.intechopen.com/books/wave-propagation-in-materialsfor-modern-applications/plasma-wave-propagation-with-light-emission-in-a-long-positive-column-discharge

\section{INTECH}

open science | open minds

\author{
InTech Europe \\ University Campus STeP Ri
}

\section{InTech China}

Unit 405, Office Block, Hotel Equatorial Shanghai 
Slavka Krautzeka 83/A

51000 Rijeka, Croatia

Phone: +385 (51) 770447

Fax: +385 (51) 686166

www.intechopen.com
No.65, Yan An Road (West), Shanghai, 200040, China 中国上海市延安西路65号上海国际贵都大饭店办公楼405单元 Phone: +86-21-62489820

Fax: $+86-21-62489821$ 
(C) 2010 The Author(s). Licensee IntechOpen. This chapter is distributed under the terms of the Creative Commons Attribution-NonCommercialShareAlike-3.0 License, which permits use, distribution and reproduction for non-commercial purposes, provided the original is properly cited and derivative works building on this content are distributed under the same license. 